\title{
Surgical treatment of low and intermediate grade lung net
}

\author{
Mariano García-Yuste ${ }^{1}$, José María Matilla ${ }^{1}$, Miguel Angel Cañizares ${ }^{2}$, Laureano Molins ${ }^{3}$, Ricardo \\ Guijarro $^{4}$; Members of the Spanish Multi-centric Study of Neuroendocrine Tumours of the Lung for the \\ Spanish Society of Pneumonology and Thoracic Surgery (EMETNE-SEPAR)
}

\begin{abstract}
${ }^{1}$ Department of Thoracic Surgery, University Clinic Hospital of Valladolid, Valladolid, Spain; ${ }^{2}$ Department of Thoracic Surgery, University Hospital of Vigo, Vigo, Spain; ${ }^{3}$ Department of Thoracic Surgery, University Clinic Hospital of Barcelona, Barcelona, Spain; ${ }^{4}$ Department of Thoracic Surgery, University General Hospital of Valencia, Valencia, Spain

Contributions: (I) Conception and design: All authors; (II) Administrative support: M García-Yuste, JM Matilla, MA Cañizares; (III) Provision of study materials or patients: All authors; (IV) Collection and assembly of data: All authors; (V) Data analysis and interpretation: M García-Yuste, JM Matilla, MA Cañizarez; (VI) Manuscript writing: All authors; (VII) Final approval of manuscript: All authors.

Correspondence to: Dr. Mariano García-Yuste. Department of Thoracic Surgery, University Clinic Hospital, Avd. Ramón y Cajal, 3, 47003 Valladolid, Spain. Email: mgyustem@gmail.com.
\end{abstract}

Background: Carcinoids now constitute complex tumours which require a multidisciplinary approach and long-term follow-up. Surgical intervention is nowadays confirmed as the mainstay of treatment.

Methods: From 1980 to 2015, EMETNE-SEPAR collected 1,339 patients treated surgically for bronchial carcinoid (1,154 typical and 185 atypical carcinoids). Standard and conservative procedures were considered with regard to surgical approach. All the patients with carcinoid were pathologically coded following the standards of the $7^{\text {th }}$ edition 2009 TNM lung cancer staging. Statistical analyses were performed in order to determine whether histology, nodal affectation and surgical technique were associated with significant differences in survival, presence of metastases and local recurrence.

Results: The influence of the surgical procedure on overall survival, the presence of metastases and local recurrence were demonstrated as no significant in our sample in central tumours $(\mathrm{P}>0.05)$. Sublobar resections in peripheral tumours are related to a decrease in survival in typical carcinoids $(\mathrm{P}=0.008)$ with nodal involvement and an increased number of recurrences in atypical carcinoids without nodal involvement $(\mathrm{P}=0.018)$.

Conclusions: In central typical carcinoid, the use of lung-sparing bronchoplastic techniques could influence local recurrence in some cases. This observation demands the intraoperative pathologic verification of an adequate surgical margin by frozen section. Peripheral typical carcinoids have been surgically treated, occasionally, by sublobar resection. However, in peripheral atypical carcinoid after a limited sublobar resection the observed increase of the probability of local recurrence makes it, in our opinion, not advisable.

Keywords: Typical carcinoid; atypical carcinoid; surgical treatment; conservative lung resection

Submitted Aug 20, 2017. Accepted for publication Sep 15, 2017.

doi: $10.21037 /$ jtd.2017.09.83

View this article at: http://dx.doi.org/10.21037/jtd.2017.09.83

\section{Introduction}

In the spectrum of neuroendocrine carcinomas the gradual deterioration in the organization of the histological pattern bears a significant relationship with the prognosis of these tumours. Typical carcinoid tumours are found at one end of the wide spectrum of these pathological entities, and at the other, small-cell neuroendocrine carcinomas. Intermediate degrees of differentiation and behaviour define the other neoplasms of this spectrum; atypical carcinoid and large cell neuroendocrine carcinoma. Based on the correlation between histologic differences and clinical prognosis of the patients, the initial classification of these tumours 
established in 1982 by the World Health Organization (WHO) (1) has been modified several times.

Carcinoid tumours are malignant and rare neoplasms. The histologic criteria for distinguishing between typical and atypical carcinoid was established by Arrigoni (2) in 1972. The 1999 WHO classification (3) has accepted stricter criteria proposed by Travis (4) for separating typical from atypical carcinoids-a reduction in the lower limit of the number of mitoses observed from 5 to 2 per $10 \mathrm{HPF}$ or the presence of punctate foci of coagulative necrosisthus defining a new histologic concept of atypical carcinoid. Based on these facts, currently, typical and atypical carcinoids-low and intermediate grade lung NETs (5) are included in the spectrum of neuroendocrine neoplasm of the lung; the gradual unstructuring of this pattern marks the histologic differences between them. Acceptance of these new classification criteria, allows us to better clarify the prognosis after treatment in typical and atypical carcinoids. Bearing in mind these facts, typical and atypical carcinoids now constitute complex tumours which require a multidisciplinary approach and long-term follow-up. Investigation of these pathological processes centres on the causes of their specific differentiation, behaviour and therapeutic possibilities.

Experience from prestigious multicentre studies (6-8) and in particular that performed by the Spanish Multicenter Study of Neuroendocrine Tumours of the Lung (EMETNE-SEPAR) in 1,339 patients treated surgically, has enabled us to ascertain the repercussions of several factors on prognosis of these types of tumours.

\section{Methods}

From 1980 to 2015, EMETNE-SEPAR collected their experience in 1,339 patients treated surgically for bronchial carcinoid. Among these patients, 1,154 had a typical carcinoid and 185 an atypical carcinoid. The histological diagnosis of typical and atypical carcinoid was confirmed by review of microscopic sections by a dedicated pathologist. All the patients with carcinoid were pathologically coded following the standards of the $7^{\text {th }}$ edition 2009 TNM lung cancer staging (9). Patients underwent surgical resection of the tumour in all cases. From 1980 to 1997, mediastinal lymph node sampling was associated, and then systematic nodal dissection was carried out.

Several variables were reviewed in all patients: age, gender, location of the tumour, size, surgical procedure, and nodal status using the TNM classification. The resection extent was also studied, dividing the series of tumours into central and peripheral.

Standard and conservative procedures were considered with regard to surgical approach. For central location, standard resection (lobectomy, bi-lobectomy, and pneumonectomy) versus bronchoplastic procedure (isolated bronchial resection and sleeve lobectomy) and for peripheral tumours standard resection versus sublobar (segmentectomy or wedge resection).

Survival analysis data was collected from a systematic follow-up database. Bearing in mind histology and nodal affectation, we studied the clinical behaviour after surgical resection of these tumours, analyzing their prognostic significance on survival and recurrence.

Statistical analysis was carried out using SPSS-21.0 statistical software package. Categorical variables were compared using Chi-squared test. Cumulative survival probabilities and comparisons were estimated by the Kaplan-Meier test. A P value of $<0.05$ was considered significant.

\section{Results}

For both typical and atypical carcinoids, the demographic details (sex and age), tumour location and tumour size are given in Table 1 .

The surgical procedures performed in patients with typical carcinoid were: standard resection, $73.1 \%$ [lobectomy 628 (54.4\%), bilobectomy 119 (10.3\%); pneumonectomy $97(8.4 \%)]$, bronchoplastic procedures $125(10.80 \%)$, sublobar resection 169 (14.6\%), and not precisely defined $16(1.4 \%)$.

For patients with atypical carcinoid, the frequency and percentage of the different surgical resections were as follows: standard resection, 80.5\% [lobectomy 103 (55.7\%), bilobectomy 16 (8.6\%); pneumonectomy 30 (16.2\%)], bronchoplastic procedures $8(4.3 \%)$, sublobar resection $19(10.3 \%)$, and not well defined $9(4.9 \%)$.

Lymph node metastases were found in 148 patients (11.05\%), of which 87 were N1 and 61 N2. Considering the histology, the distribution of cases was: $59 \mathrm{~N} 1(5.1 \%)$ and $31 \mathrm{~N} 2(2.7 \%)$ in typical carcinoids, and $28 \mathrm{~N} 1$ (15.1\%) and $30 \mathrm{~N} 2(16.2 \%)$ in atypical carcinoids.

In typical carcinoid, 42 out of 1,154 patients (3.6\%) presented some kind of recurrence during the outcome: 8 corresponded to loco-regional recurrences $(0.7 \%)$, 28 were distance metastases (2.4\%), and 6 patients had both types $(0.5 \%)$. Among the patients with atypical carcinoid 
Table 1 Demographic and tumoural characteristics of patients with typical and atypical carcinoid tumours

\begin{tabular}{lcc}
\hline Characteristics & Typical carcinoid & Atypical carcinoid \\
\hline Sex (\%) & & 51.9 \\
Men & 43.8 & 48.1 \\
Women & 52.6 & 54.8 \\
Mean age (years) & 50.6 & $21-80$ \\
Range & $4-82$ & \\
Location (\%) & & 55.2 \\
Central & 65.5 & 44.8 \\
Peripheral & 34.5 & 31.6 \\
Mean tumoural size (mm) & 25.1 & $9-105$ \\
Range & $1-99$ & \\
\hline
\end{tabular}

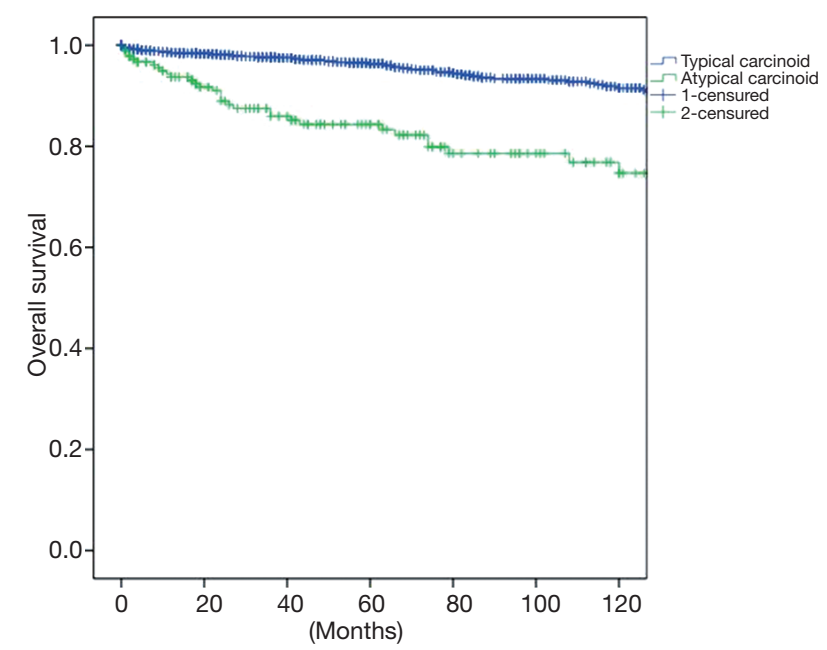

Figure 1 Kaplan-Meier survival and statistical comparative analysis for patients with typical and atypical carcinoid tumours.

patients, 40 (20.54\%) had tumour recurrence, 28 (15.1\%) presented metastases, 5 patients $(2.7 \%)$ had local recurrence and 5 patients $(2.7 \%)$ both.

The analysis of mortality during follow-up in patients with typical carcinoid shows that 15 of the 1,154 patients (1.3\%) died due to tumour recurrence and 53 (4.6\%) due to an independent cause. When the same analysis was performed in patients with atypical carcinoid, 16 of the 185 patients $(8.6 \%)$ died due to tumour recurrence and $17(9.1 \%)$ due to an independent cause

The overall survival at 5 and 10 years was in typical and

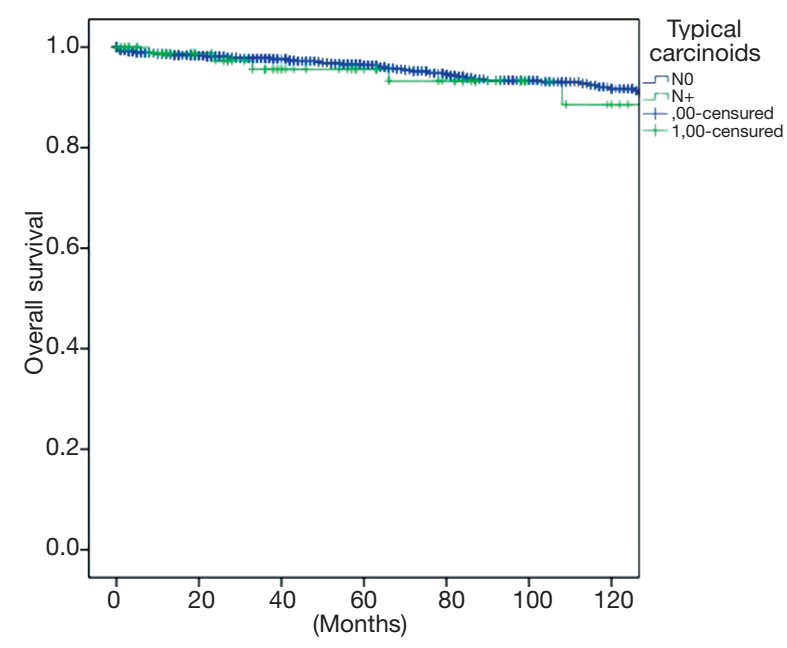

Figure 2 Prognostic influence of $\mathrm{N}$-status in typical carcinoid [univariate analysis (Kaplan Meier)].

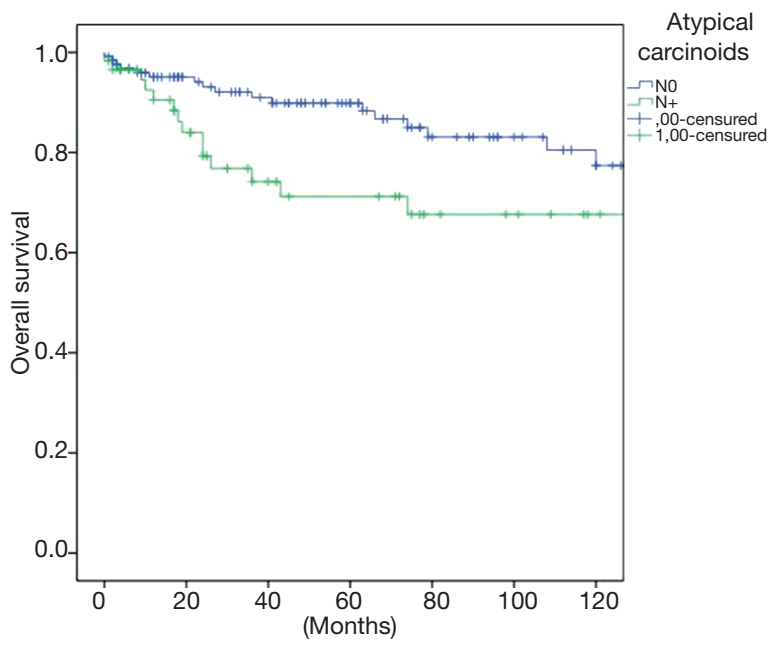

Figure 3 Prognostic influence of $\mathrm{N}$-status in atypical carcinoid [univariate analysis (Kaplan Meier)].

atypical carcinoids $96.3 \%$ and $91.5 \%, 84.3 \%$ and $74.7 \%$ respectively $(\mathrm{P}=0.000)$ (Figure 1). When the influence of nodal involvement on survival in both groups of patients was analyzed, a significant statistical difference was found between the patients with atypical carcinoid: typical N0: 96\% and $91 \% ; \mathrm{N}+: 84 \%$ and $74 \%(\mathrm{P}=0.486)$ (Figure 2); and atypical carcinoids N0: $90 \%$ and $77 \% ; \mathrm{N}+71 \%$ and $67 \%$ ( $\mathrm{P}=0.033)$ (Figure 3).

Considering central versus peripheral location and nodal involvement, analysis of the influence of the surgical procedure on the presence of metastases; overall survival 
Table 2 Influence of surgical procedure and nodal involvement in overall survival and the presence of metastases and local recurrence considering central vs. peripheral location in these tumours

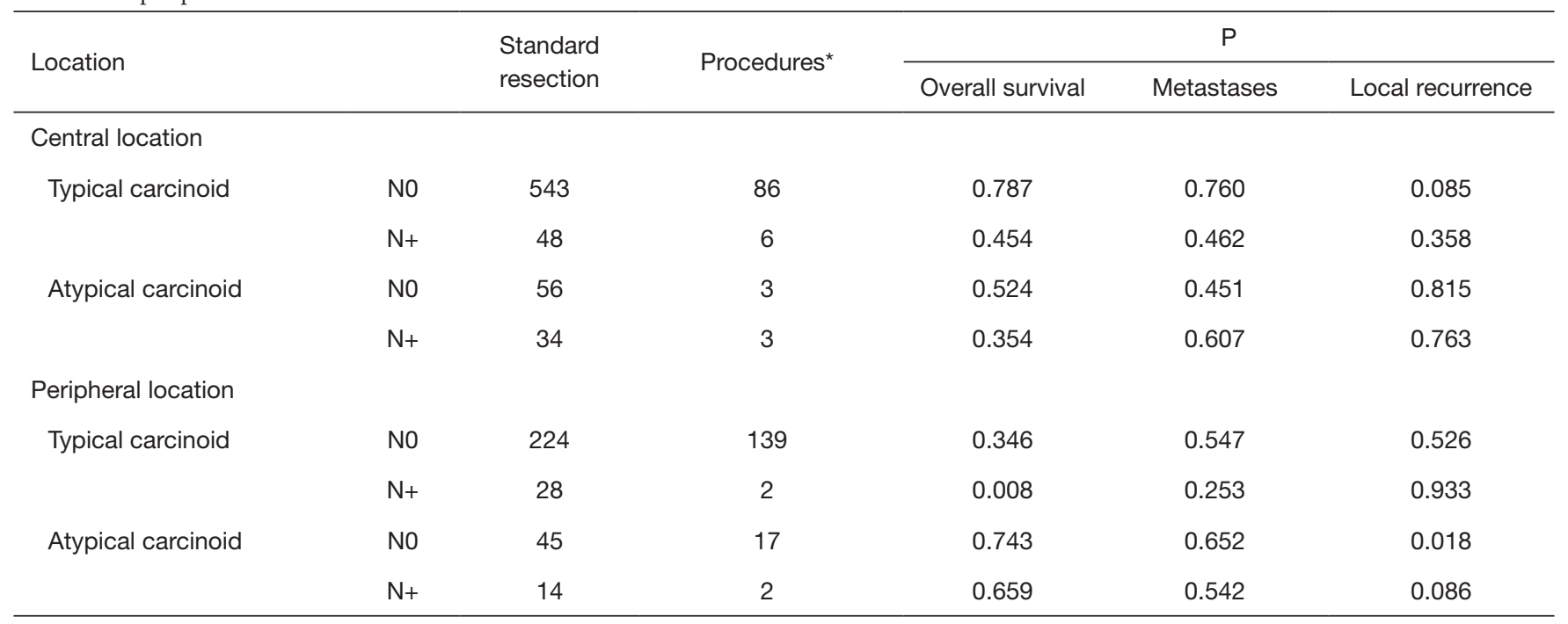

*, Bronchoplastic procedures for central location and sublobar resection for peripheral location.

and local recurrence were demonstrated as non-significant in our sample of central tumours. Sublobar resections in peripheral tumours are related to a decrease in survival in typical carcinoids with nodal involvement and an increased number of recurrences in atypical carcinoids without nodal involvement (Table 2).

\section{Discussion}

Because carcinoid tumours can invade and metastasize, both typical and atypical carcinoids are currently classified as malignant. The typical carcinoid behaves less aggressively, it rarely relapses after complete surgical resection, and nodal involvement and metastases to distant sites are rare. The atypical carcinoid represents, in the spectrum of neuroendocrine lung tumours, an intermediate stage between typical carcinoids and large and small cell neuroendocrine carcinomas. In addition to these facts, that surgical intervention is confirmed as the mainstay of treatment.

As has been observed in previous studies $(10,11)$, the increase in the number of patients analyzed enables us to confirm with accuracy the relationship between the increase in mean age and histologic degradation in these types of tumours. Variations in percentage of incidence of these tumours in patients of one or other sex are also significant. In our experience, the typical carcinoid affects female patients with a frequency significantly above that of atypical carcinoid.
The predominance of central tumours was noteworthy in carcinoid tumours, with peripheral location percentages slightly higher in atypical versus typical. These facts allow us to correlate peripheral tumour location with a worse prognosis for our patients. A significant difference in tumour size was found between typical and atypical carcinoid in our patients. This fact has also been previously recorded by us and other authors and reaffirms an increase in size as the level of histologic deterioration progresses $(12,13)$.

In lung cancer, tumour size and the involvement of lymph nodes are the local anatomic factors with the greatest influence on prognosis. Their classification into different degrees and the establishment of stages provide an adequate understanding of the behaviour of the tumour and the possibilities for treating them $(14,15)$. As in other bronchogenic carcinomas, in carcinoid tumours lymph node involvement is a factor of high prognostic value and its impact is marked by the histological type. Nodal disease is a frequent feature in AC. In fact, its relationship with prognosis was reported in these patients $(16,17)$.

Following these criteria, according to the Union Internationale Contre le Cancer/American Joint Committee on Cancer (UICC/AJCC) (18) the seventh edition published of the TNM system (9) has been recommended for the classification of pulmonary carcinoid tumours, although a histologic distinction between typical carcinoid versus $\mathrm{AC}$ was not generally made in the data analyzed for the International Association for the Study of Lung Cancer 
(IASLC) staging project. For these reasons and in consensus with the recently published ENETS recommendations (8), it will be necessary to refine descriptive categories according to tumour size, multicentricity, or other components of T, $\mathrm{N}$, and $\mathrm{M}$ factors, for these also to be meaningful in PCs (IASLC Lung Cancer Perspective Staging Project).

In our experience, nodal involvement in atypical carcinoids is significantly higher than that observed in typical carcinoids. Moreover, their ability to produce distant metastasis is greater than in cases of typical carcinoid. Investigation for lymph node metastases seems to be an unavoidable requisite to establish prognosis and to evaluate therapeutic strategies $(7,12)$, taking into account the results presented and the few studies that have analysed in-depth the impact of lymph node involvement on the prognosis of bronchial carcinoids. However, having studied the repercussions of this factor on prognosis, we should consider the following points when deciding the therapeutic approach: (I) the importance of histologic aggressiveness as a determining factor for nodal involvement in these tumours; (II) the definition of the sensitivity of PET/CT, SPECT in-pentetreotide or PET/CT with Ga-DOTATOC for detecting nodal involvement; (III) the need for preoperative histological confirmation of nodal involvement by EBUS/EUS or video mediastinoscopy biopsy specimen; (IV) the appropriateness of neo-adjuvant treatment when nodal mediastinal involvement, mainly in atypical carcinoids, is detected preoperatively; (V) the need, both in typical and atypical carcinoids-irrespective of their central or peripheral location-to always perform a regulated mediastinal nodal dissection; (VI) the frequency of recurrence after surgical treatment, clearly different for typical and atypical carcinoids, especially when there is nodal involvement; (VII) the implication of lymph node involvement in the decision to use lung-sparing bronchoplastic techniques in central carcinoids or limited sublobar resection in peripheral atypical carcinoids.

Surgery is the treatment of choice for pulmonary carcinoids with the aim of removing the tumour and preserving as much lung tissue as possible. The surgical approach is dependent on the size, location, and tissue type. An adequate lung resection and a complete mediastinal nodal dissection are mandatory. The extent of lymph node management should conform to the IASLC recommendations for the certainty factor in the designation of $\mathrm{R} 0$ resection; this involves a minimum of six nodes/ stations, three of which should be mediastinal including the subcarinal station (19).
Following these oncologic norms, a conservative bronchoplastic lung resection could be considered in typical and atypical carcinoid central tumours avoiding pneumonectomy. In the presence of distant pneumonitis and destroyed lung parenchyma, an initial local endobronchial resection to disobliterate the airway may be undertaken for drainage before re-assessment for lung parenchymalsparing surgery (15). Nevertheless, as Detterbeck states (16), institutions vary in the use of lung-sparing bronchoplastic techniques for central tumours and sublobar resections for peripheral tumours. Experience from prestigious multicentre studies and in particular that gained by EMETNE in patients treated surgically, has enabled us to ascertain the repercussions on the prognosis of these types of surgical approach. Given the results, we agree with other authors that in central typical carcinoid, the use of lung-sparing bronchoplastic techniques could influence local recurrence in some cases. This observation demands the intraoperative pathologic verification of an adequate surgical margin by frozen section. Resection margins should be indicated, measuring the distance from the tumour edge to guarantee radical excision, on surgical specimens (20).

The appropriateness of performing sublobar resections in peripheral lung cancer tumours has been widely debated for some time $(21,22)$. In recent years, various studies have highlighted the superiority of lobectomy over segmentectomy in terms of prognostic outcome; the indication for segmental lung resection being essentially reserved for patients with impaired lung function, peripheral tumours, stage Ia cancer, and always associated with complete mediastinal nodal dissection. Peripheral typical carcinoids have been surgically treated, occasionally, by broad wedge resection or segment resection. However, in peripheral atypical carcinoid after a limited sublobar resection, the increased probability observed of local recurrence makes it, in our opinion, not advisable. In our experience, the increased study patient sample size has merely served to corroborate this. Therefore, today it is generally accepted that the standards of bronchopulmonary carcinoma treatment must always be fulfilled and the best results are achieved after operations such as lobectomy. In patients with limited pulmonary function, if a lobectomy is not possible, standard segment resection achieves better results than broad wedge resection (23).

In the aforementioned situations, progress in the diagnosis and treatment of the listed outcomes demands a knowledge of the number of patients surgically treated for these types of tumours per year. The limitations of the 
study are given both by the limited particularization in papers of the number of patients treated per year during a specific period and by the scarce multi-centres studies with a sufficient number of patients. The experience gathered by multicentre studies could be an example to illustrate the average flow of patients treated per year for the two different types of pulmonary carcinoid tumours.

\section{Acknowledgements}

None.

\section{Footnote}

Conflicts of Interest: The authors have no conflicts of interest to declare.

Ethical Statement: EMETNE-SEPAR possesses the approval of the ethical committees of the hospitals that participate in the study. The patients sign an informed assent in order that his information is introduced in an anonymous database.

\section{References}

1. The World Health Organization histological typing of lung tumours. Second edition. Am J Clin Pathol 1982;77:123-36.

2. Arrigoni MG, Woolner LB, Bernatz PE. Atypical carcinoid tumors of the lung. J Thorac Cardiovasc Surg 1972;64:413-21.

3. Travis WD, Sobin LH. Histologic typing of lung and pleural tumours international histologic classification of tumours. New York: Springer-Verlag, 1999.

4. Travis WD, Rush W, Flieder DB, et al. Survival analysis of 200 pulmonary neuroendocrine tumors with clarification of criteria for atypical carcinoid and its separation from typical carcinoid. Am J Surg Pathol 1998;22:934-44.

5. Travis WD, Brambilla E, Nicholson AG, et al. The 2015 World Health Organization Classification of Lung Tumors: Impact of Genetic, Clinical and Radiologic Advances Since the 2004 Classification. J Thorac Oncol 2015;10:1243-60.

6. Filosso PL, Guerrera F, Evangelista A, et al. Prognostic model of survival for typical bronchial carcinoid tumours: analysis of 1109 patients on behalf of the European Association of Thoracic Surgeons (ESTS) Neuroendocrine Tumours Working Group. Eur J Cardiothorac Surg 2015;48:441-7; discussion 447 .
7. Filosso PL, Rena O, Guerrera F, et al. Clinical management of atypical carcinoid and large-cell neuroendocrine carcinoma: a multicentre study on behalf of the European Association of Thoracic Surgeons (ESTS) Neuroendocrine Tumours of the Lung Working Groupt. Eur J Cardiothorac Surg 2015;48:55-64.

8. Caplin ME, Baudin E, Ferolla P, et al. Pulmonary neuroendocrine (carcinoid) tumors: European Neuroendocrine Tumor Society expert consensus and recommendations for best practice for typical and atypical pulmonary carcinoids. Ann Oncol 2015;26:1604-20.

9. Goldstraw P. International Association for the Study of Lung Cancer Staging Manual in Thoracic Oncology. Florida, USA: Editorial Rx Press, 2009.

10. García-Yuste M, Matilla JM, Cueto A, et al. Typical and atypical carcinoid tumours: analysis of the experience of the Spanish Multi-centric Study of Neuroendocrine Tumours of the Lung. Eur J Cardiothorac Surg 2007;31:192-7.

11. Davini F, Gonfiotti A, Comin C, et al. Typical and atypical carcinoid tumours: 20-year experience with 89 patients. J Cardiovasc Surg (Torino) 2009;50:807-11.

12. Rea F, Rizzardi G, Zuin A, et al. Outcome and surgical strategy in bronchial carcinoid tumors: single institution experience with 252 patients. Eur J Cardiothorac Surg 2007;31:186-91.

13. García-Yuste M, Matilla JM, González-Aragoneses F. Neuroendocrine tumors of the lung. Curr Opin Oncol 2008;20:148-54.

14. Cardillo G, Sera F, Di Martino M, et al. Bronchial carcinoid tumors: nodal status and long-term survival after resection. Ann Thorac Surg 2004;77:1781-5.

15. Lim E, Yap YK, De Stavola BL, et al. The impact of stage and cell type on the prognosis of pulmonary neuroendocrine tumors. J Thorac Cardiovasc Surg 2005;130:969-72.

16. Detterbeck FC. Management of carcinoid tumors. Ann Thorac Surg 2010;89:998-1005.

17. Lim E, Goldstraw P, Nicholson AG, et al. Proceedings of the IASLC International Workshop on Advances in Pulmonary Neuroendocrine Tumors 2007. J Thorac Oncol 2008;3:1194-201.

18. Travis WD, Giroux DJ, Chansky K, et al. The IASLC Lung Cancer Staging Project: proposals for the inclusion of broncho-pulmonary carcinoid tumors in the forthcoming (seventh) edition of the TNM Classification for Lung Cancer. J Thorac Oncol 2008;3:1213-23.

19. Rusch VW, Asamura H, Watanabe H, et al. The IASLC lung cancer staging project: a proposal for a new 
international lymph node map in the forthcoming seventh edition of the TNM classification for lung cancer. J Thorac Oncol 2009;4:568-77.

20. Öberg K, Hellman P, Ferolla P, et al. Neuroendocrine bronchial and thymic tumors: ESMO Clinical Practice Guidelines for diagnosis, treatment and follow-up. Ann Oncol 2012;23 Suppl 7:vii120-3.

21. Ginsberg RJ, Rubinstein LV. Randomized trial of lobectomy versus limited resection for T1 N0 non-small

Cite this article as: García-Yuste M, Matilla JM, Cañizares MA, Molins L, Guijarro R; Members of the Spanish Multicentric Study of Neuroendocrine Tumours of the Lung for the Spanish Society of Pneumonology and Thoracic Surgery (EMETNE-SEPAR). Surgical treatment of low and intermediate grade lung net. J Thorac Dis 2017;9(Suppl 15):S1435-S1441. doi: 10.21037/jtd.2017.09.83 cell lung cancer. Lung Cancer Study Group. Ann Thorac Surg 1995;60:615-22; discussion 622-3.

22. Kent M, Landreneau R, Mandrekar S, et al. Segmentectomy versus wedge resection for non-small cell lung cancer in high-risk operable patients. Ann Thorac Surg 2013;96:1747-54; discussion 1754-5.

23. Yendamuri S, Gold D, Jayaprakash V, et al. Is sublobar resection sufficient for carcinoid tumors? Ann Thorac Surg 2011;92:1774-8; discussion 1778-9. 
The Spanish Multicenter Study of Neuroendocrine Tumors of the Lung of the Spanish Society of Pneumonology and Thoracic Surgery (EMETNE-SEPAR)

Mariano García-Yuste, MD PhD, Jose-Maria Matilla, MD PhD, and Henar Borrego, MD(University Hospital, Valladolid); Ignacio Escobar, MD and Joan Moya, MD, $\mathrm{PhD}$ (Bellvitge Hospital, Barcelona); David Saldaña, MD PhD and Pilar Garrido, MD (Ramon-y-Cajal Hospital, Madrid); Francisco Cerezo, MD and Javier Algar, MD (Reina-Sofia Hospital, Cordoba); Federico GonzalezAragoneses, MD, Carlos Simon, MD, Emilio Alvarez, MD and Maria Cebollero, MD (Gregorio-Marañon Hospital, Madrid); Jorge Cerezal Garrido, MD (University Hospital, Alicante); Ricardo Guijarro, MD, PhD and Antonio Arnau, MD, PhD (General-University Hospital, Valencia); Luis Lopez-Rivero, MD, PhD, Santiago Quevedo, MD and Maria-Carmen Camacho, MD (Insular Hospital, Las-Palmas); Javier Perez, MD (Germans-TriasPujol Hospital, Badalona); Laureano Molins, MD (Clinic Hospital, Barcelona); Jose-Manuel Mier (Sagrado-Corazón Hospital, Barcelona); Antonio Cueto, MD, Abel SanchezPalencia, MD and Angel Concha, MD (Virgen-de-las-
Nieves Hospital, Granada); Jorge Freixinet, $\mathrm{MD}, \mathrm{PhD}$, Pedro Rodriguez, MD and Teresa Romero, MD (DrNegrin Hospital, Las Palmas); Juan Torres, MD, PhD and Juan Bermejo, MD (Virgen-Arrixaca Hospital, Murcia); Nicolas Moreno, MD, PhD and Ana Blanco, MD (VirgenRocio Hospital, Sevilla); Mercedes de-la-Torre, MD and AnaCapdevilla, MD (University Hospital, Coruña); Ramon Moreno, MD (La-Princesa Hospital, Madrid); Mireia Serra and Ramon Rami-Porta, MD (Mutua-Terrassa Hospital, Terrassa); Ricardo Arrabal, MD, and Antonio Benitez, MD (Carlos-Haya Hospital, Malaga); Andres Varela, MD and Mar Cordoba, MD (Puerta-Hierro-Majadahonda Hospital, Madrid); Miguel-Angel Cañizares MD, PhD, Eva GarciaFontan, MD, Montserrat Blanco-Ramos, MD, $\mathrm{PhD}$ and Ana Gonzalez-Piñeiro, MD (University Hospital, Vigo); Ignacio Muguruza, MD, PhD and José Zapatero, MD, $\mathrm{PhD}$, (Fundación-Jiménez-Díaz Hospital, Madrid); JuanJose Rivas and MD, Patricia Menal, MD (Miguel-Servet Hospital, Zaragoza); Genaro Galan, MD, PhD (Clinic Hospital, Valencia); and Emilio Ansotegui, MD (La-Fe Hospital, Valencia). 